\title{
Physical Nucleon Form Factors From Lattice QCD
}

Phys.Rev.C71:055204,2005; ArXiv:[nucl-th/0508049 ]

Hrayr H. Matevosyan,

Louisiana State University / JLab.

Anthony W. Thomas,

Thomas Jefferson Laboratory.

Gerald A. Miller,

University of Washington, Seattle. 


\section{The Plan.}

- Introduction.

- Light Front Cloudy Bag Model.

- Fits to the lattice data and extrapolations to the physical region.

- Conclusions. 


\section{Introduction.}

- The electromagnetic form factors of the nucleon are an invaluable source of information on its structure.

- The recent advances in the lattice QCD provide us with form factor calculations over wide range of momentum transfer.

Problems:

$>$ Extrapolation of the lattice calculations to the physical quark mass region.

$>$ Extrapolation of $\mathrm{a} \rightarrow 0, \mathrm{~V} \rightarrow \infty$.

$>$ Unquenching. 


\section{Motivation.}

\section{Three problems to address:}

- Does the model describe lattice data?

- Using the model to extrapolate the Form Factors to large values of $Q^{2}$ for lattice values of $m_{\pi}$.

- Using the model to extrapolate the Form Factors to the physical $m_{\pi^{*}}$. 


\section{The Light Front Cloudy Bag Model (LFCBM). [(. Mier, Phys. Rev. C 66, 032201(R) (2002)]}

\section{The key features:}

- Respects Lorentz Invariance.

- Respects Chiral Symmetry.

- Incorporates pion-cloud effects.

- Reproduces the four nucleon electromagnetic form factors.

- Is easily adaptable for calculations in high quark mass ("lattice") regime. 


\section{LFCBM Wave-Function.}

In light-front dynamics four-vectors are

$$
\begin{gathered}
\left(p^{+}, p^{-}, p^{\perp}\right), p^{ \pm}=p^{0} \pm p^{3}, p^{\perp}=\left(p^{1}, p^{2}\right) ; \\
p^{\mu} p_{\mu}=m^{2} \rightarrow p^{-}=\left(\left(p^{\perp}\right)^{2}+m^{2}\right) / p^{+} .
\end{gathered}
$$

-is similar to nonrelativistic kinetic energy form.

The bare (pionless) nucleon wave function:

$$
\Psi\left(p_{i}\right)=\Phi\left(M_{0}^{2}\right) u\left(p_{1}\right) u\left(p_{2}\right) u\left(p_{3}\right) \psi\left(p_{1}, p_{2}, p_{3}\right)
$$

$\psi$ - spin-isospin amplitude, $u\left(p_{i}\right)$ - Dirac spinors, $\Phi$ - momentum distribution wave function.

$\Phi$ is taken from F. Schlumpf, [arXiv:hep-ph/9211255].

$$
\begin{gathered}
\Phi\left(M_{0}\right)=N\left(M_{0}^{2}+\beta^{2}\right)^{\gamma} \\
M_{0}^{2}=\frac{K_{\perp}^{2}}{\eta(1-\eta)}+\frac{k_{\perp}^{2}+M^{2}}{\eta \xi(1-\xi)}+\frac{M^{2}}{1-\eta}
\end{gathered}
$$

where $M$ is the constituent quark mass (assumed constant). 


\section{Nucleon EM Form Factors.}

\section{The Quark Core Contribution:}

In the Drell-Yan frame $\left(\mathrm{q}^{+}=0\right)$, using light-front spinors

$$
F_{1}\left(Q^{2}\right)=\left\langle N, \uparrow\left|J^{+}\right| N, \uparrow\right\rangle, \quad \text { and } \quad Q F_{2}\left(Q^{2}\right)=\left(-2 M_{N}\right)\left\langle N, \uparrow\left|J^{+}\right| N, \downarrow\right\rangle \text {. }
$$

\section{Including The Pion Cloud:}

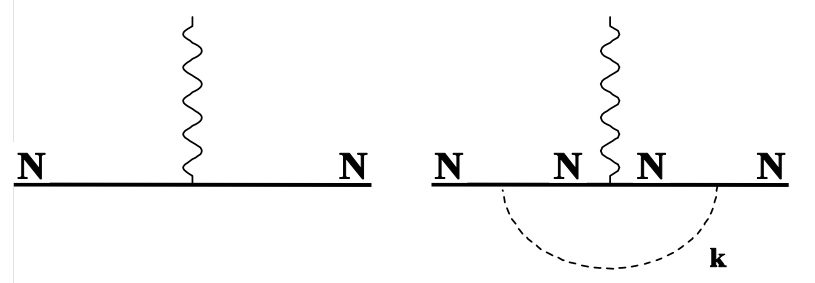

(a)
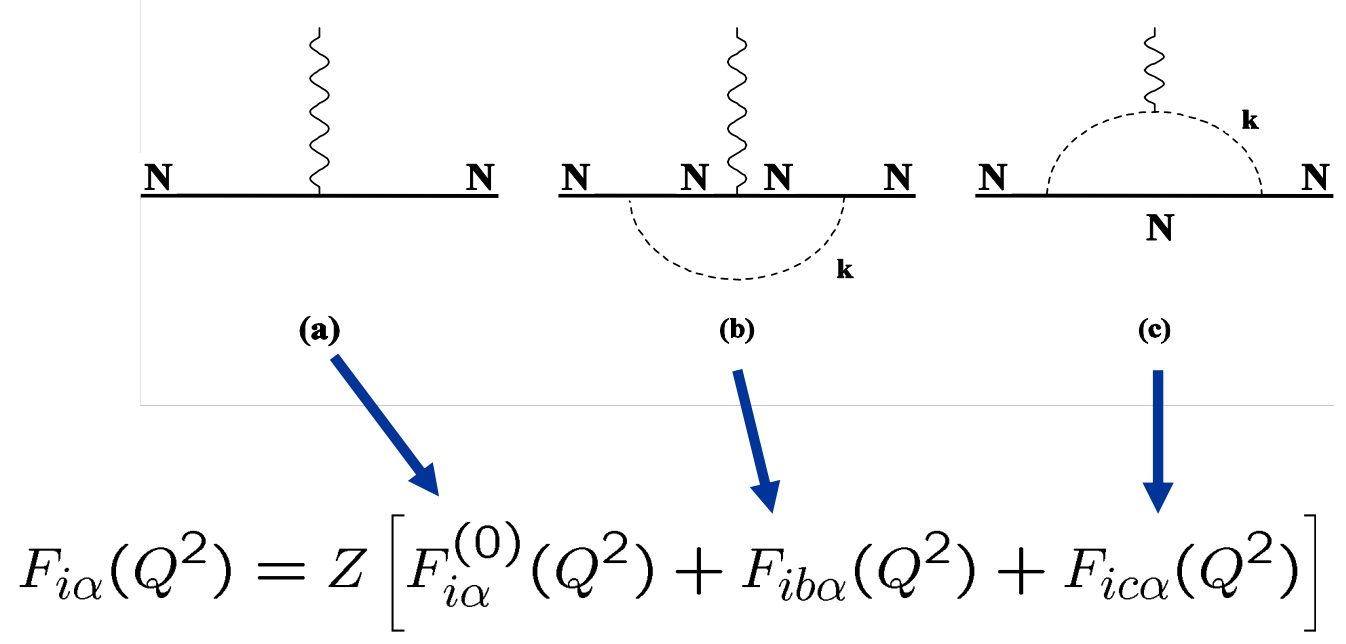


\section{$>$ Lattice Data Set.}

We fit the form factor calculations by QCDSF Collaboration.

[M. Gockeler et al., Phys.Rev.D71:034508,2005.]

The calculations were carried out using quenched Clover fermions.

The data range:

$>a-\{0.093 \mathrm{fm}, 0.067 \mathrm{fm}, 0.051 \mathrm{fm}\}$,

$\mathrm{V}-\left\{16^{3} \times 32, \quad 24^{3} \times 48, \quad 32^{3} \times 48\right\}$,

$>\mathrm{m}_{\pi} \sim\{1.2 \mathrm{GeV}, 0.6 \mathrm{GeV}\}$,

$>\mathrm{M}_{\mathrm{N}} \sim\{2 \mathrm{GeV}, 1.5 \mathrm{GeV}\}$,

$>\mathrm{Q}^{2} \sim\left\{0.6 \mathrm{GeV}^{2}, 2.3 \mathrm{GeV}^{2}\right\}$. 


\section{Fitting Parameters.}

\section{The Model Parameters:}

- $\mathbf{M}$ - the Constituent Quark Mass

- $\beta$ - the Nucleon Momentum Distribution WF Parameter - INSENSITIVE AT HIGH PION MASS

- $\gamma$ - the Nucleon Momentum Distribution WF Parameter

- $\Lambda$ - the Pion-Nucleon Form Factor Parameter - INSENSITIVE AT HIGH PION MASS

We need to extrapolate the Constituent Quark Mass

$$
M=M_{\chi}+\frac{c m_{q}^{\text {phys }}}{\left(m_{\pi}^{\text {phys }}\right)^{2}} m_{\pi}^{2}
$$

$\mathrm{cm}_{\mathrm{q}}$ phys $=5.9 \mathrm{MeV}$, and $\mathrm{M} \chi$ is the constituent quark mass in the chiral limit. [I. Cloet et. al., Phys. Rev. C 65, 062201 (2002)]

\section{Our Fitting Parameters:}

- $\quad \gamma$ is varied for each $m_{\pi}$.

- $\mathrm{M} \chi$ is varied for each lattice a. 


\section{Extrapolation to high $\mathrm{Q}^{2}$ of calculated $F F$ for different $m_{\pi}$ for $a=0.051 \mathrm{fm}$.}
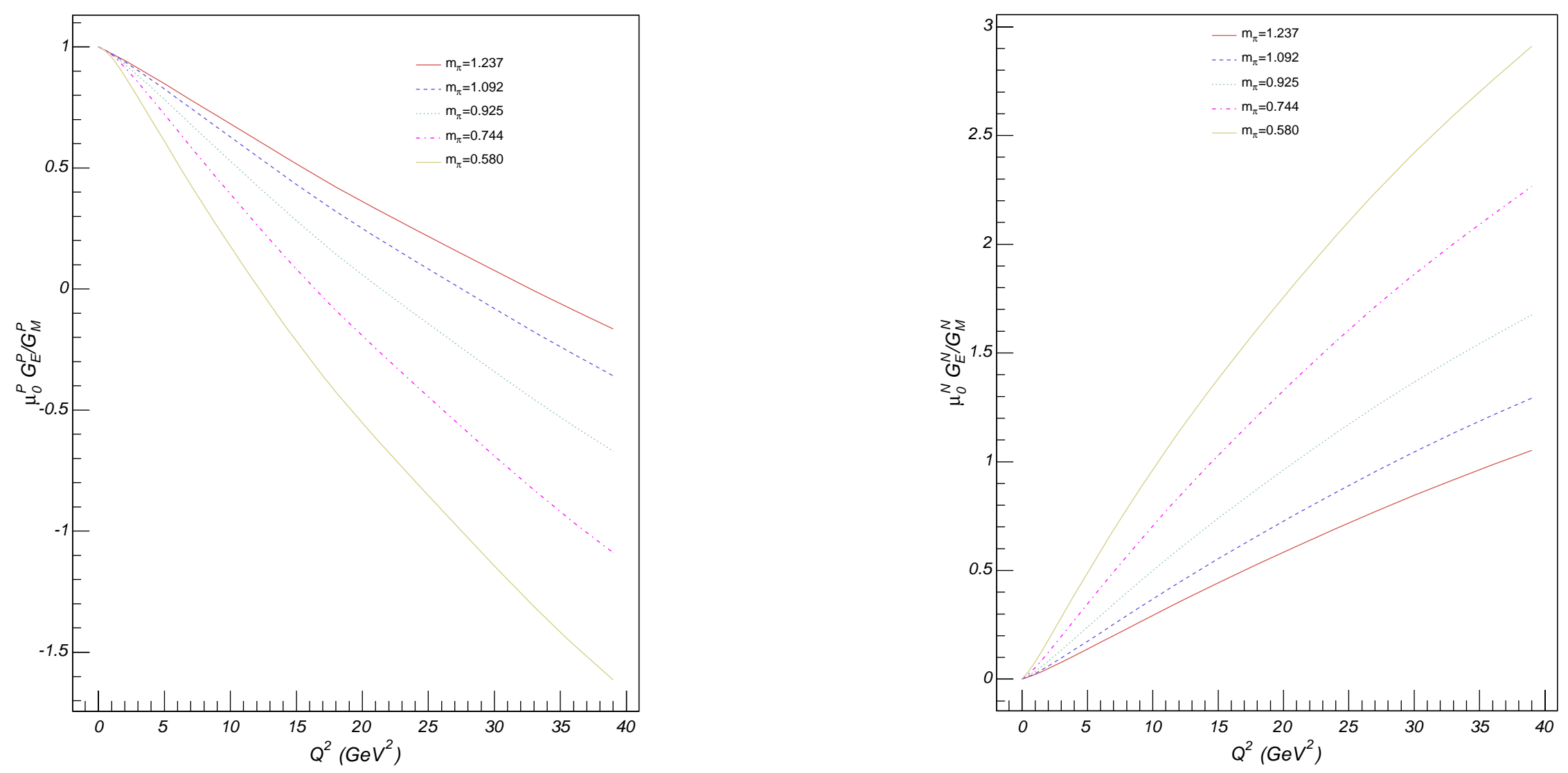


\section{Extrapolation of the fitting parameters for $a=0.051 \mathrm{fm}$.}
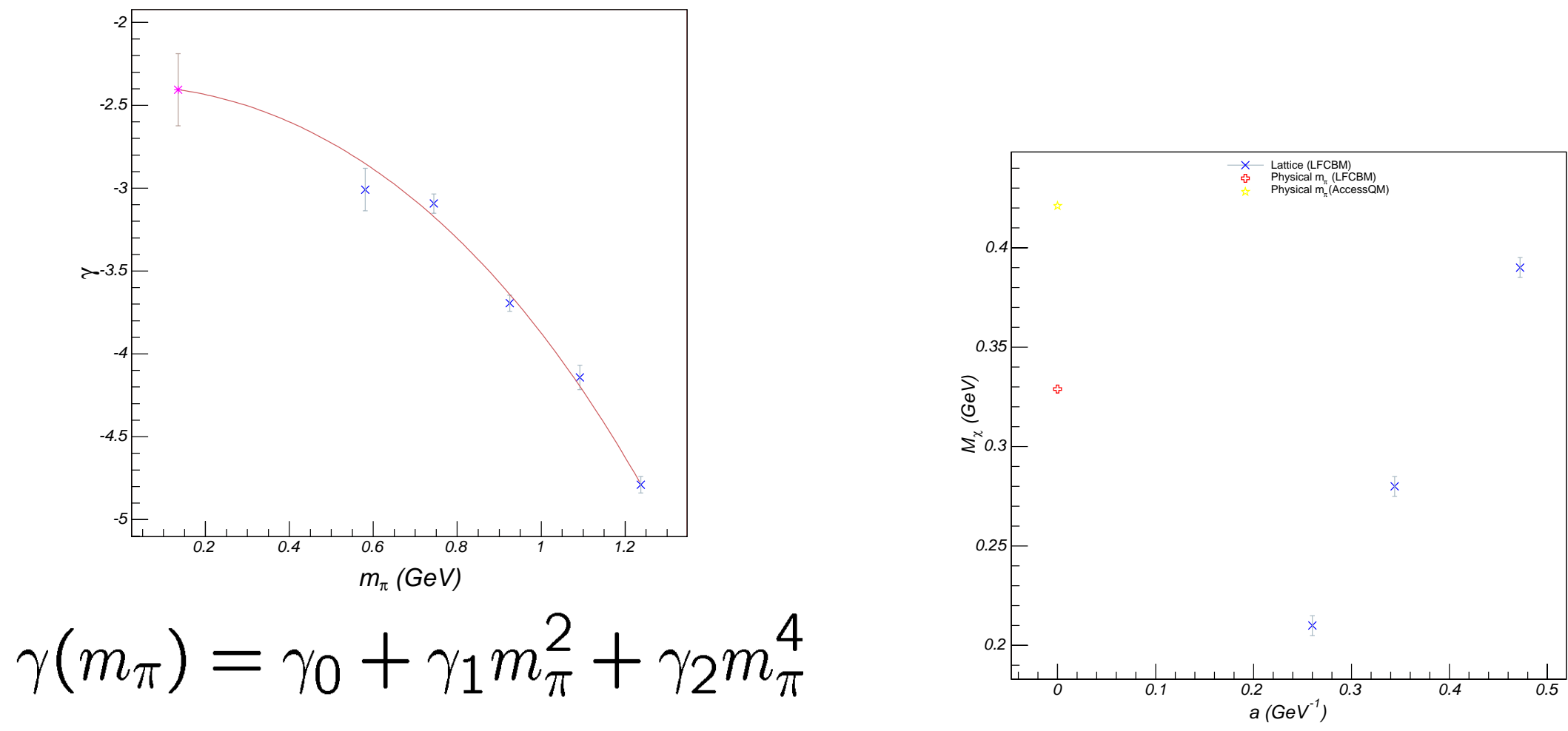


\section{Physical Nucleon FF from LFCBM extrapolation for $a=0.051 \mathrm{fm}$.}

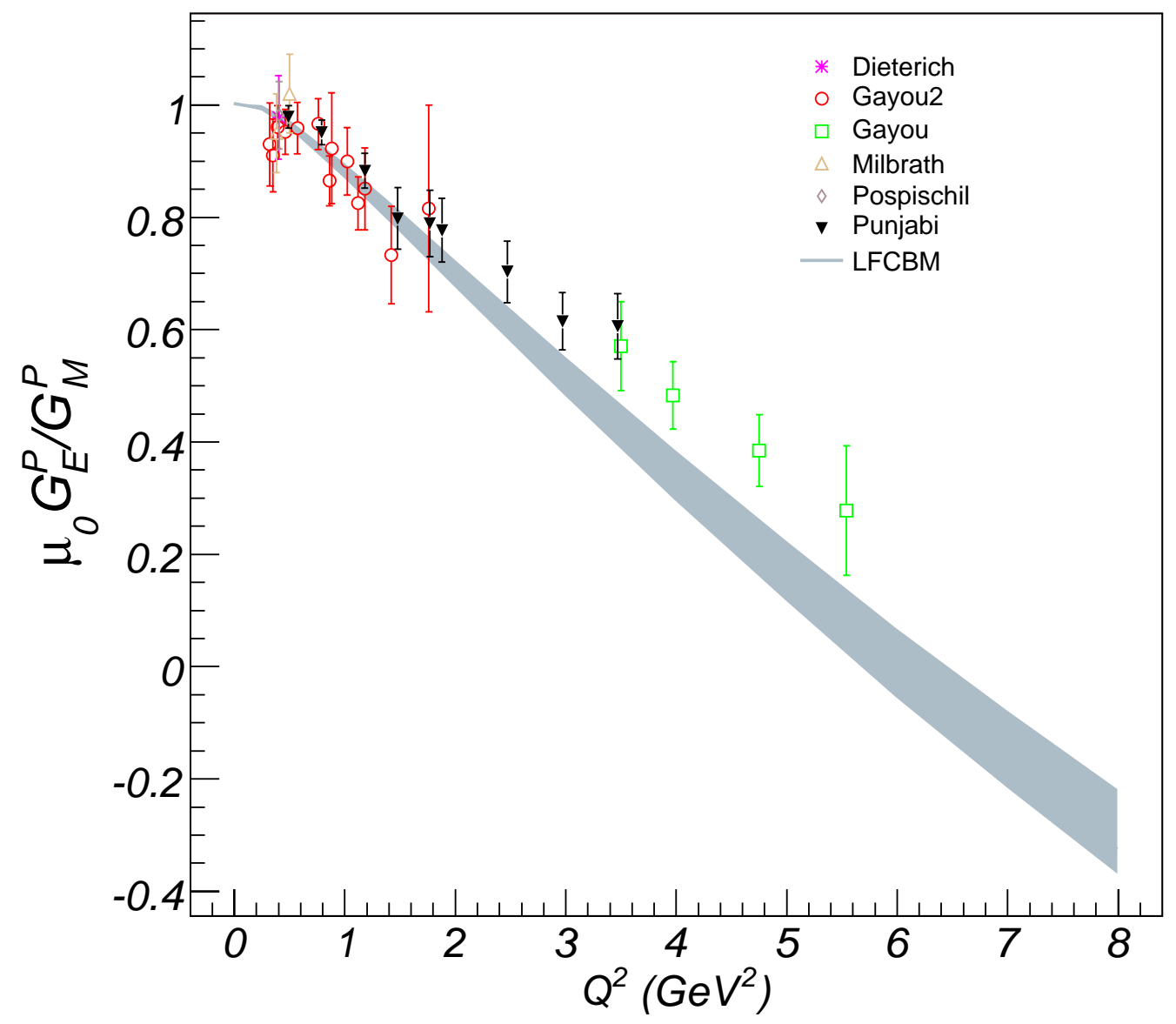




\section{Conclusion.}

We have employed the Light Front Cloudy Bag Model (LFCBM) for nucleon form factor calculations.

- LFCBM gives quite accurate description of the lattice data points. It was used to both extrapolate in $\mathrm{Q}^{2}$ the calculated form factors, and with smooth quadratic extrapolation of the fitting parameters vs. $\mathrm{m}_{\pi}$ to extrapolate the form factors to the physical quark mass region.

* We foresee the lattice calculated form factors as an alternative test of validity for any model of the nucleon structure.

* As new more accurate data at lower $\mathrm{m}_{\pi}$ and higher $\mathrm{Q}^{2}$ data will be available in the future, one will be able to have stricter tests and more accurate extrapolations to the physical mass region. 


\section{Nucleon EM Form Factors.}

\section{Dirac $F_{\mathbf{1}}$ and Pauli $F_{\mathbf{2}}$ form factors:}

$$
\left\langle N, \lambda^{\prime} p^{\prime}\left|J^{\mu}\right| N, \lambda p\right\rangle=\bar{u}_{\lambda^{\prime}}\left(p^{\prime}\right)\left[F_{1}\left(Q^{2}\right) \gamma^{\mu}+\frac{F_{2}\left(Q^{2}\right)}{2 M_{N}} i \sigma^{\mu \nu}\left(p^{\prime}-p\right)_{\nu}\right] u_{\lambda}(p)
$$

\section{Sachs Form Factors:}

$$
\begin{aligned}
& G_{E}=F_{1}-\frac{Q^{2}}{4 M_{N}^{2}} F_{2}, \\
& G_{M}=F_{1}+F_{2} .
\end{aligned}
$$




\section{The Quark Core Contribution.}

The evaluation of the form factors is simplified by using the so-called Drell-Yan reference frame in which $q^{+}=0$, so $Q^{2}=q_{\perp}{ }^{2}=q_{1}{ }^{2}, K^{\prime}{ }_{\perp}=K_{\perp}-\eta q_{\perp}$. If light-front spinors for the nucleons are used, the form factors can be expressed in terms of matrix elements of the plus component of the current:

$$
F_{1}\left(Q^{2}\right)=\left\langle N, \uparrow\left|J^{+}\right| N, \uparrow\right\rangle, \quad \text { and } \quad Q F_{2}\left(Q^{2}\right)=\left(-2 M_{N}\right)\left\langle N, \uparrow\left|J^{+}\right| N, \downarrow\right\rangle .
$$

The form factors are calculated using the "good" component of the current, $\mathrm{J}^{+}$, to suppress the effects of quark-pair terms.

$$
\begin{aligned}
& F_{1}^{(0)}\left(Q^{2}\right)=\int \frac{d^{2} k_{\perp} d \xi}{\xi(1-\xi)} \frac{d^{2} K_{\perp} d \eta}{\eta(1-\eta)} \tilde{\Phi}^{\dagger}\left(M_{0}^{\prime}\right) \widetilde{\Phi}\left(M_{0}\right) \times\left\langle\chi_{0}^{r e l}\left(\mathbf{p}_{1}^{\prime}, \mathbf{p}_{2}^{\prime}\right) \mid \chi_{0}^{r e l}\left(\mathbf{p}_{1}, \mathbf{p}_{2}\right)\right\rangle\left\langle\uparrow \mathbf{p}_{3}^{\prime} \mid \uparrow \mathbf{p}_{3}\right\rangle \\
& \frac{Q F_{2}^{(0)}\left(Q^{2}\right)}{2 M_{N}}=\int \frac{d^{2} k_{\perp} d \xi}{\xi(1-\xi)} \frac{d^{2} K_{\perp} d \eta}{\eta(1-\eta)} \widetilde{\Phi}^{\dagger}\left(M_{0}^{\prime}\right) \tilde{\Phi}\left(M_{0}\right) \times\left\langle\chi_{0}^{r e l}\left(\mathbf{p}_{1}^{\prime}, \mathbf{p}_{2}^{\prime}\right) \mid \chi_{0}^{r e l}\left(\mathbf{p}_{1}, \mathbf{p}_{2}\right)\right\rangle\left\langle\uparrow \mathbf{p}_{3}^{\prime} \mid \downarrow \mathbf{p}_{3}\right\rangle
\end{aligned}
$$




\section{Including the Pion Cloud.}

$$
\begin{aligned}
& \frac{\{\mathbf{N}}{\mathbf{N} \frac{\mathbf{N}}{\mathbf{N}} \mathbf{N}} \frac{\mathbf{N}_{\mathrm{N}}}{\mathbf{N}} \\
& \text { (a) } \\
& F_{i \alpha}\left(Q^{2}\right)=Z\left[F_{i \alpha}^{(0)}\left(Q^{2}\right)+F_{i b \alpha}\left(Q^{2}\right)+F_{i c \alpha}\left(Q^{2}\right)\right] \\
& F_{1 b n}\left(Q^{2}\right)=g_{0}^{2} \int_{0}^{1} d \alpha \alpha \int \frac{d^{2} L}{(2 \pi)^{3}} R_{N}\left(\mathrm{~L}^{(+)^{2}}, \alpha\right) R_{N}\left(\mathrm{~L}^{(-)^{2}}, \alpha\right)\left[\left(F_{1 p}^{(0)}\left(Q^{2}\right)+F_{1 n}^{(0)}\left(Q^{2}\right) / 2\right)\left(\alpha^{2}\left(M^{2}-Q^{2} / 4\right)+L^{2}\right)\right. \\
& \left.\left.-\left(F_{2 p}^{(0)}\left(Q^{2}\right)+F_{2 n}^{(0)}\left(Q^{2}\right) / 2\right)\right)\left(\alpha^{2} Q^{2} / 2\right)\right], \\
& F_{2 b n}\left(Q^{2}\right)=-g_{0}^{2} \int_{0}^{1} d \alpha \alpha \int \frac{d^{2} L}{(2 \pi)^{3}} R_{N}\left(\mathrm{~L}^{(+)^{2}}, \alpha\right) R_{N}\left(\mathrm{~L}^{(-)^{2}}, \alpha\right)\left[\left(F_{1 p}^{(0)}\left(Q^{2}\right)+\frac{1}{2} F_{1 n}^{(0)}\left(Q^{2}\right)\right)\left(2 \alpha^{2} M^{2}\right)\right. \\
& \left.\left.+\left(F_{2 p}^{(0)}\left(Q^{2}\right)+\frac{1}{2} F_{2 n}^{(0)}\left(Q^{2}\right)\right)\right)\left(\alpha^{2} M^{2}\left(1-Q^{2} / 4 M^{2}\right)+\left(L_{x}^{2}-L_{y}^{2}\right)\right)\right]
\end{aligned}
$$




\section{Including the Pion Cloud.}

$F_{1 c n}\left(Q^{2}\right)=-g_{0}^{2} F_{\pi}\left(Q^{2}\right) \int_{0}^{1} d \alpha \alpha \int \frac{d^{2} K}{(2 \pi)^{3}} R\left(\mathrm{~K}^{(+)^{2}}, \alpha\right) R\left(\mathrm{~K}^{(-)^{2}}, \alpha\right)\left[K^{2}+M^{2} \alpha^{2}-(1-\alpha)^{2} \frac{Q^{2}}{4}\right]$

$F_{2 c n}\left(Q^{2}\right)=-g_{0}^{2}\left(2 M^{2}\right) F_{\pi}\left(Q^{2}\right) \int_{0}^{1} d \alpha \alpha^{2}(1-\alpha) \int \frac{d^{2} K}{(2 \pi)^{3}} R\left(\mathrm{~K}^{(+)^{2}}, \alpha\right) R\left(\mathrm{~K}^{(-)^{2}}, \alpha\right)$

where $\mathrm{g}_{0}$ is the bare $\pi \mathrm{N}$ coupling constant, and the renormalized coupling constant

$$
\begin{aligned}
& Z g_{0}^{2}=g^{2} / 4 \pi=13.5 \\
& \mathbf{L}_{\perp}^{( \pm)} \equiv \mathbf{L}_{\perp} \pm \alpha \mathbf{q}_{\perp} / 2, \alpha \equiv \frac{k^{+}}{p^{+}} \\
& D_{N}\left(k_{\perp}^{2}, \alpha\right) \equiv M^{2} \alpha^{2}+k_{\perp}^{2}+\mu^{2}(1-\alpha) \\
& R_{N}\left(k_{\perp}^{2}, \alpha\right) \equiv \frac{F_{\pi N}^{N}\left(k_{\perp}^{2}, \alpha\right)}{D_{N}\left(k_{\perp}^{2}, \alpha\right)}, \mathbf{K}_{\perp}^{( \pm)} \equiv \mathbf{K}_{\perp} \pm(1-\alpha) \mathbf{q}_{\perp} / 2 .
\end{aligned}
$$

The $\pi \mathrm{N}$ form factor is taken as [A. Dobrovolskaya, K. Ter-Martirosian, V. Zoller , Z.Phys.C53:263-268,1992 ]

$$
F_{\pi N}\left(k_{\perp}^{2}, \alpha\right)=e^{-\left(D_{N}\left(k_{\perp}^{2}, \alpha\right) / 2 \alpha(1-\alpha) \wedge^{2}\right)},
$$




\section{Proton $\mu_{P} G_{E} / G_{M}$.}

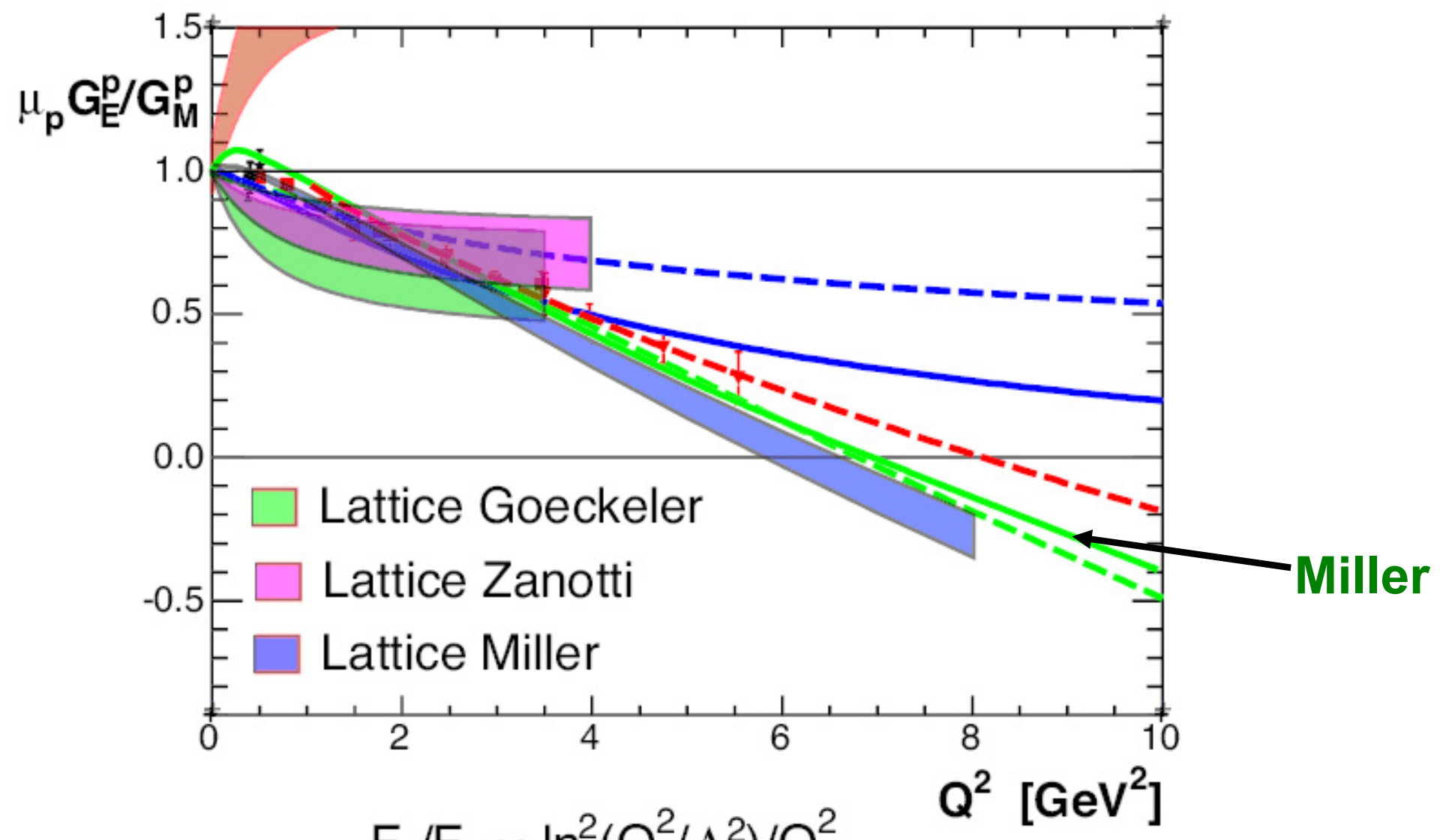

---- $F_{2} / F_{1} \propto \ln ^{2}\left(Q^{2} / \Lambda^{2}\right) / Q^{2}$

Kees de Jager 


\section{Adaptation of LFCBM to the lattice regime.}

The LFCBM is defined by choosing four free parameters: $M, \beta, \gamma, \mathrm{L}$ so as to best reproduce the four experimentally measured electromagnetic form factors of the nucleon.

The pion masses used in the lattice calculation are very large, and the resulting pion-loop contribution is very small. Therefore the value of $L$ could not be determined from lattice data and its value was held fixed at $L=0.58 \mathrm{GeV}$ taken from $\mathrm{G}$. Miller's fits to experimental data.

Varying $\beta$ did not change the description of the lattice data significantly, so it was held fixed at $\beta=0.607 \mathrm{GeV} / \mathrm{c}$ taken from Schlumpf's work. 


\section{The best-fit values of the parameters.}

TABLE I. Lattice data and LFCBM fitting parameters.(All expressed in powers of $\mathrm{GeV}$.)

\begin{tabular}{lllll}
\hline \hline$a$ & $m_{\pi}$ & $M_{N}$ & $M_{\chi}$ & $\gamma$ \\
\hline \hline 0.47 & 1.146 & 2.062 & $0.390(5)$ & $-6.12(7)$ \\
0.47 & 1.068 & 1.981 & $0.390(5)$ & $-5.67(6)$ \\
0.47 & 0.873 & 1.746 & $0.390(5)$ & $-4.95(9)$ \\
0.47 & 0.752 & 1.567 & $0.390(5)$ & $-4.78(12)$ \\
0.47 & 0.638 & 1.503 & $0.390(5)$ & $-4.67(15)$ \\
0.47 & 0.135 & 0.938 & $0.390(5)$ & $-4.79(46)$ \\
\hline 0.34 & 1.201 & 2.141 & $0.280(5)$ & $-5.03(6)$ \\
0.34 & 1.035 & 1.933 & $0.280(5)$ & $-4.37(5)$ \\
0.34 & 0.881 & 1.732 & $0.280(5)$ & $-4.99(5)$ \\
0.34 & 0.706 & 1.522 & $0.280(5)$ & $-3.51(6)$ \\
0.34 & 0.135 & 0.938 & $0.280(5)$ & $-2.91(29)$ \\
\hline 0.26 & 1.237 & 2.202 & $0.210(5)$ & $-4.78(5)$ \\
0.26 & 1.092 & 2.028 & $0.210(5)$ & $-4.14(7)$ \\
0.26 & 0.925 & 1.802 & $0.210(5)$ & $-3.69(5)$ \\
0.26 & 0.744 & 1.600 & $0.210(5)$ & $-3.09(6)$ \\
0.26 & 0.580 & 1.379 & $0.210(5)$ & $-3.01(13)$ \\
0.26 & 0.135 & 0.938 & $0.210(5)$ & $-2.41(22)$ \\
\hline
\end{tabular}




\section{Physical Neutron FF from LFCBM extrapolation for $a=0.26 \mathrm{GeV}-1$.}
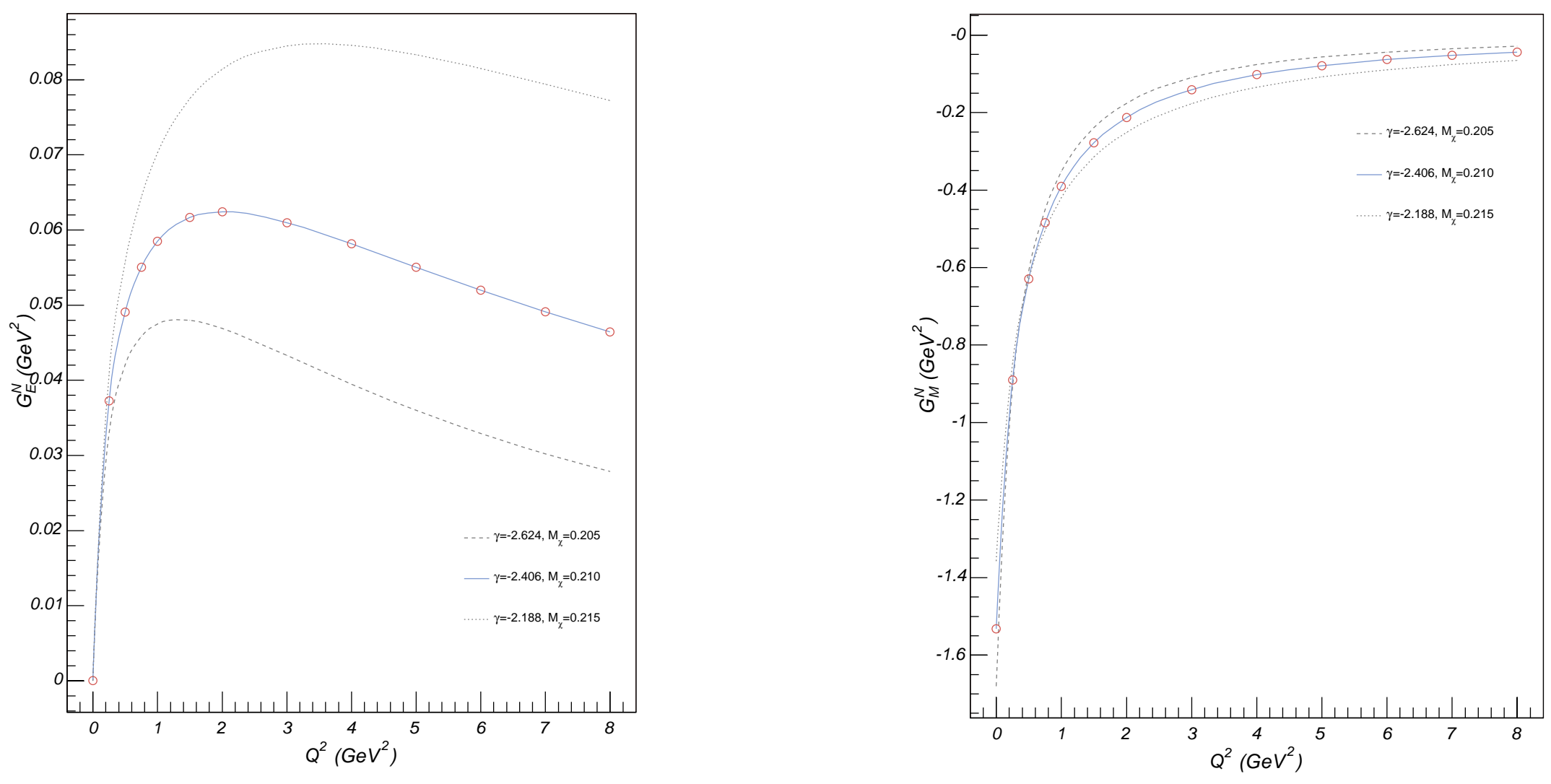


\section{Physical Nucleon FF from LFCBM extrapolation for $a=0.26 \mathrm{GeV}-1$.}
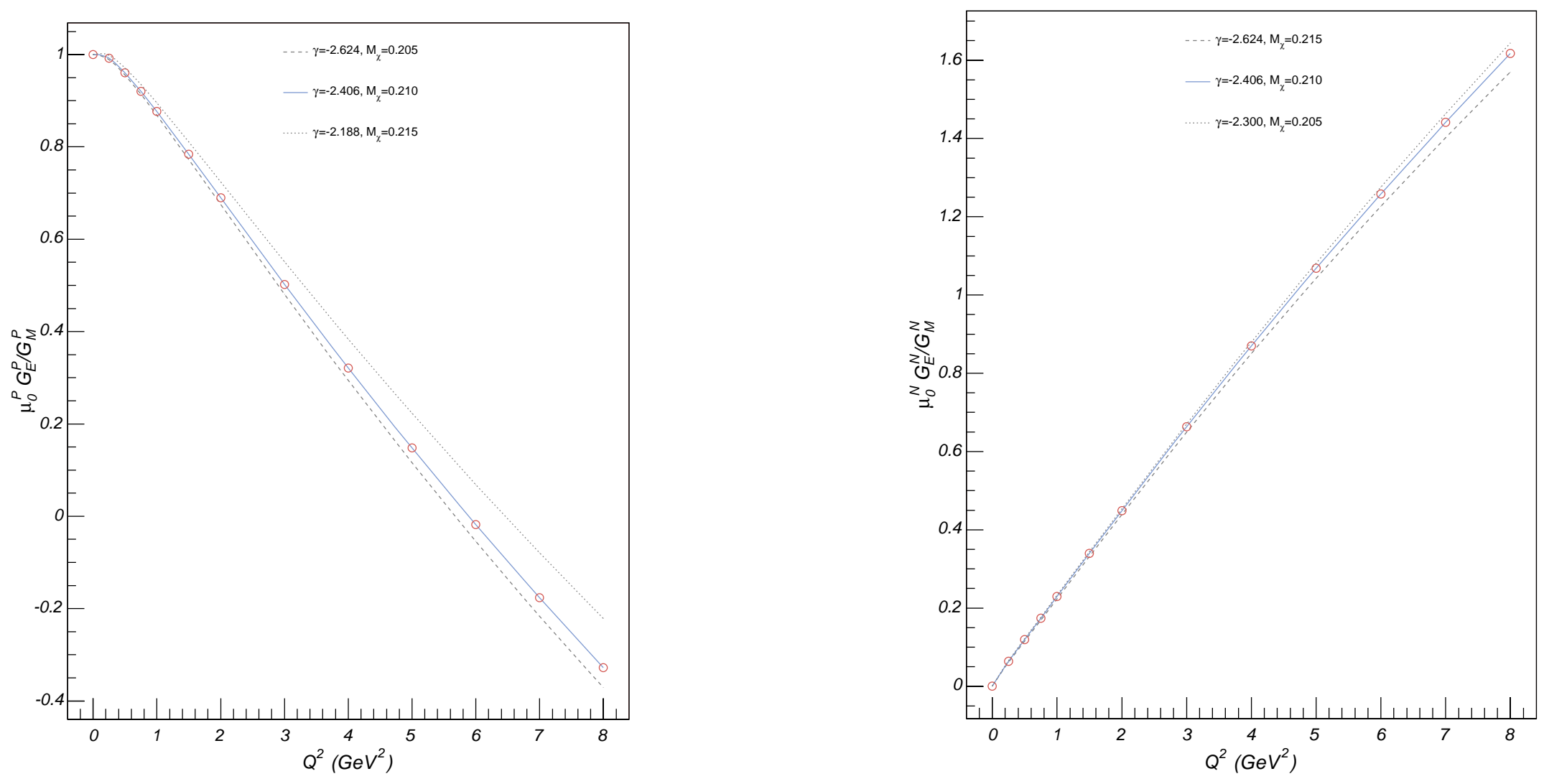


\section{Physical Proton FF from LFCBM extrapolation for $a=0.26 \mathrm{GeV}-1$.}
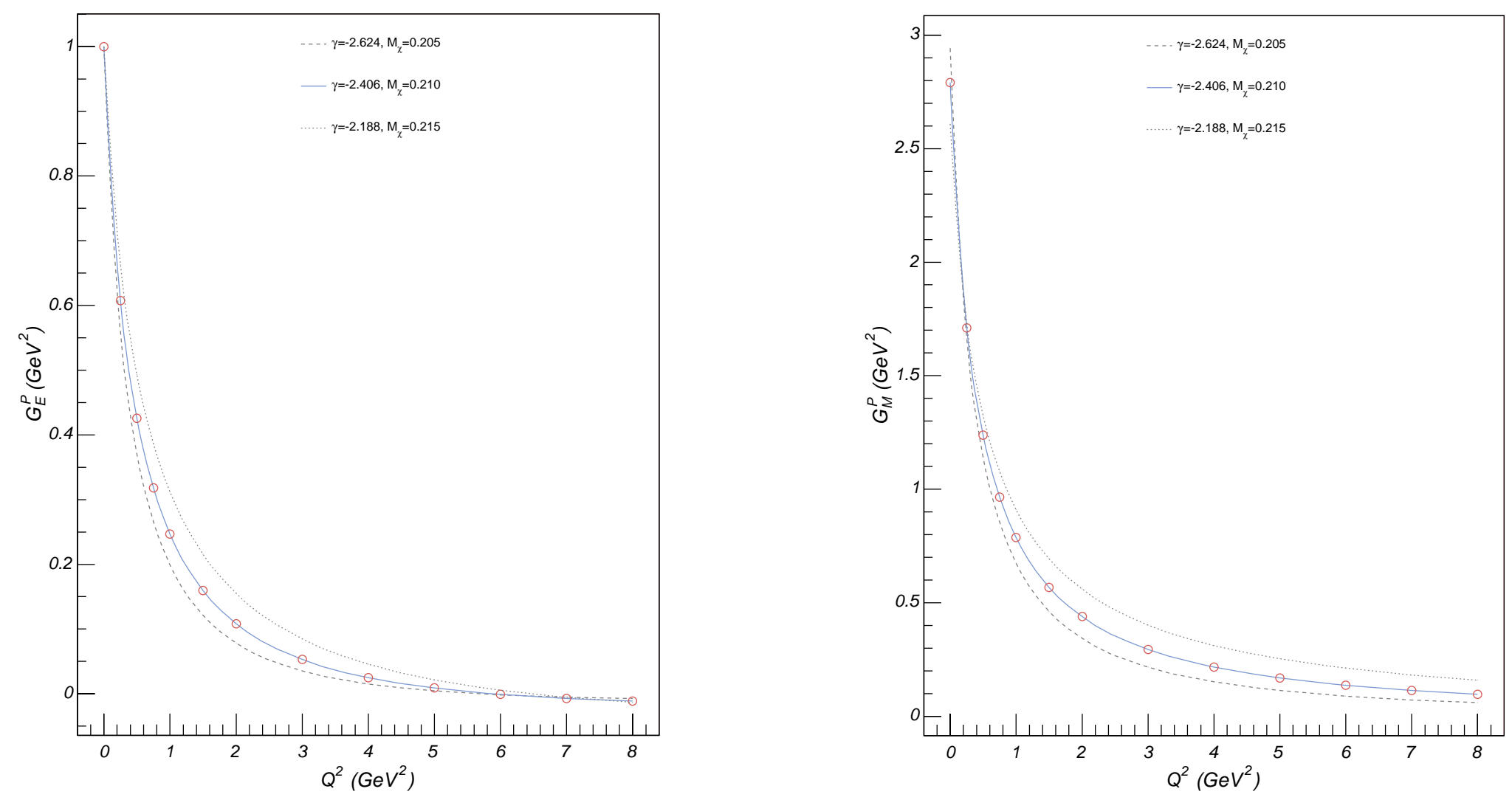


\section{Model (GKex)}

[E. Lomon, Phys. Rev. C66: 045501, 2002]

The key features:

- Is a generalized VMD model

- Predictions of nucleon $\mathrm{G}_{E}, \mathrm{G}_{\mathrm{M}}$ are in good agreement with the experiment.

- The high- $\mathrm{Q}^{2}$ behavior of the calculated form factors agrees with the PQCD prediction.

- Is easily adaptable for calculations in high quark mass ("lattice") regime. 


\section{The GKex model.}

In Gari-Krümpelmann model the "soft" photons couples to the "vector meson cloud" of the nucleon, while "hard" photons penetrate deep and couple to the "quark core" of the nucleon. The scale is set by a set of cut-off masses, which are found by fits to the data.

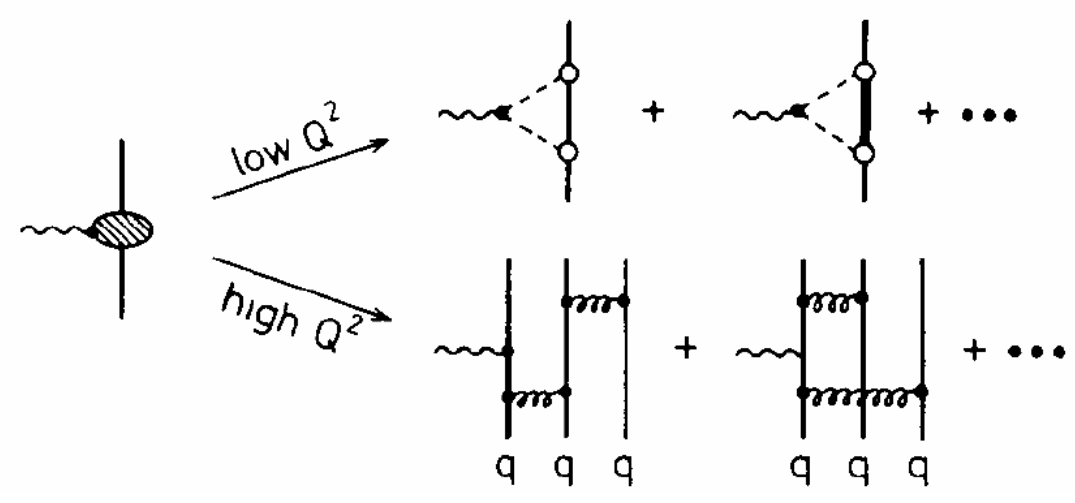

M. Gari and W. Krumpelmann, Phys. Lett. B173, 10 (1986).

In the GKex pole contributions of the $\omega(782), \phi(1020), \omega^{0}(1420), \rho(770)$ and $\rho^{0}(1450)$ mesons were included in order to achieve a good fit to the experimental data available. 


\section{The GKex model.}

Fits to the experimental data points were made using different sets of data, some of which excluded the controversial high proton $G_{E} / G_{M}$ measured previously by Rosenbluth separation method. The fits with different data sets were labeled accordingly: GKex(01), GKex(01-), GKex(02S) and GKex(02L).

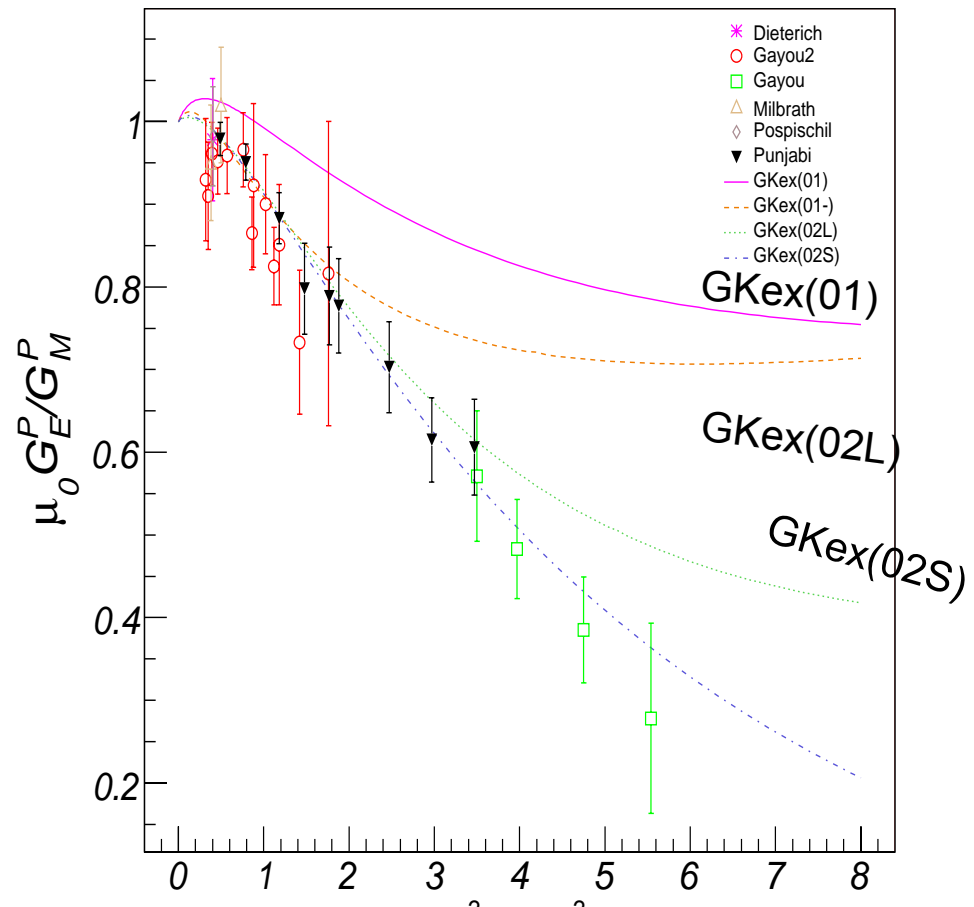

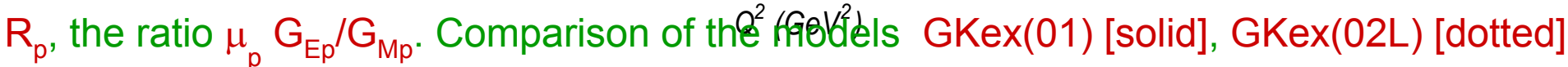




\section{Fits to the lattice proton form}

\section{factors.}
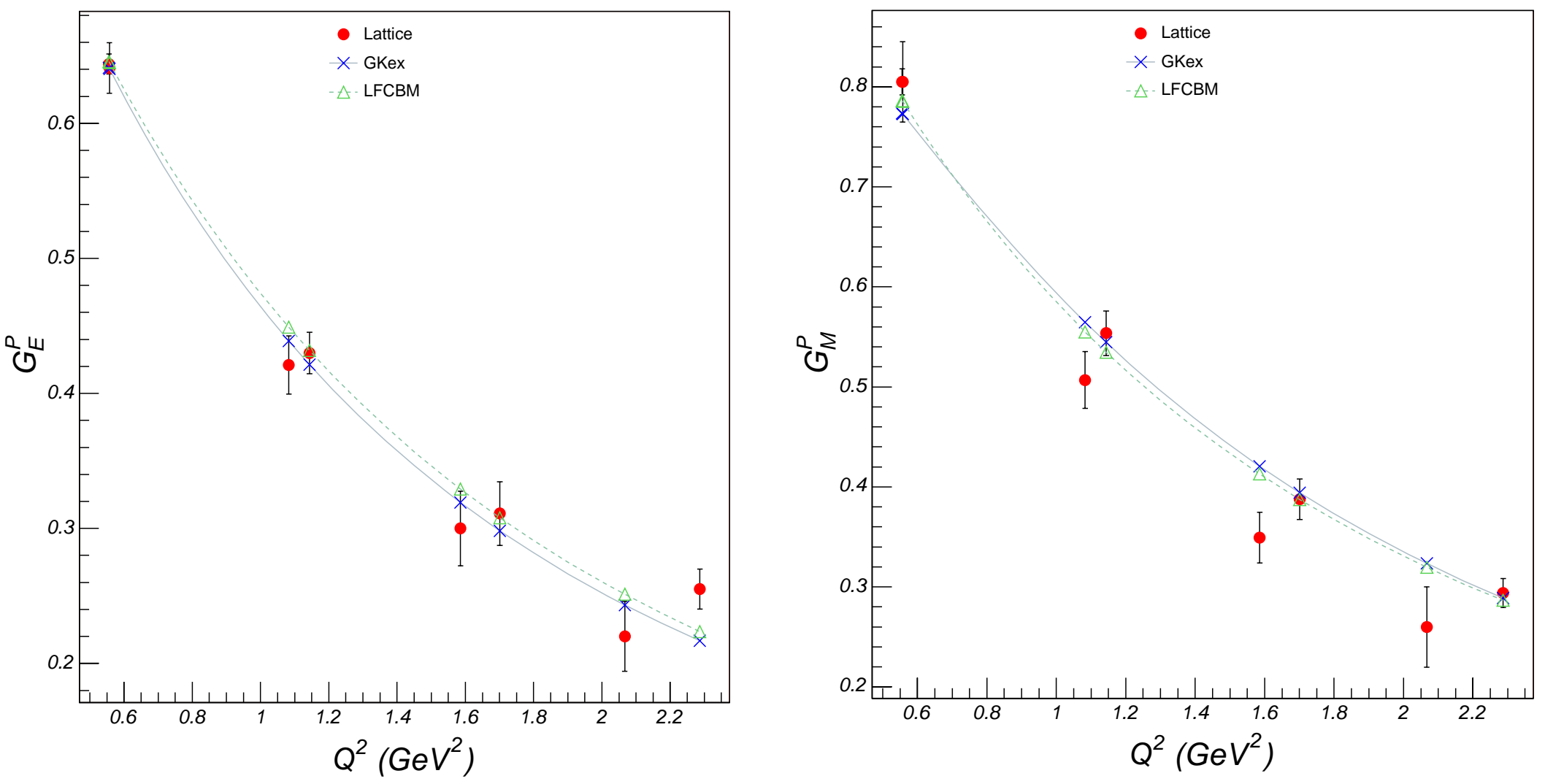

GKex(01-) fit [solid] to QCDSF data for proton GE (in units of e) and GM (in units of $\mathrm{e} / 2 \mathrm{MN}$ ) for a lattice spacing $\mathrm{a}=0.26 \mathrm{GeV}-1, \mathrm{MN}=2.20 \mathrm{GeV}$ and $\mathrm{m} \pi=1.24 \mathrm{GeV}$. LFCBM fits [dashed] are also shown for a comparison. 


\section{Table of the fitting parameters.}

The resulting fitting parameters for all versions of GKex for the 170 fitting lattice data points of lattice spacing $a=0.26 \mathrm{GeV}^{-1}$ are presented in the table below. The $\chi^{2}=81$ for the LFCBM fit to the same data set.

TABLE I. GKex fitting parameters and $\chi^{2}$ for lattice spacing $a=0.26 \mathrm{GeV}^{-1}$.

\begin{tabular}{|c|c|c|c|c|}
\hline \multicolumn{3}{|c|}{ Gkex(01) Gkex(01-) } & \multicolumn{2}{|c|}{ Gkex(02L) Gkex(02S) } \\
\hline$\chi^{2}$ & 185 & 103 & 671 & 217 \\
\hline$a_{1}^{I V}$ & $-1.80(16)$ & -2.35 & $-1.7(2)$ & $-1.86(17)$ \\
\hline$b_{1}^{I V}$ & $0.46(11)$ & 0.81 & $0.54(14)$ & $0.39(13)$ \\
\hline $\mathrm{a}_{2}^{I V}$ & $-11.9(5)$ & -55.2 & $-1(1)$ & $-10.9(5)$ \\
\hline $\mathrm{b}_{2}^{I V}$ & $2.98(36)$ & 18 & $-0.34(76)$ & $2.34(38)$ \\
\hline$a_{1}^{I S}$ & $-0.99(18)$ & -1.61 & $-0.39(9)$ & $-0.58(1)$ \\
\hline$b_{1}^{I S}$ & $-0.21(14)$ & 0.28 & $0.06(6)$ & $-0.078(73)$ \\
\hline$a_{2}^{I S}$ & $8.6(18)$ & 31 & $2.53(58)$ & $-0.32(14)$ \\
\hline $\mathrm{b}_{2}^{I S}$ & $2.6(14)$ & -0.44 & $0.62(41)$ & $0.1(1)$ \\
\hline$a^{\Lambda}$ & $0.034(38)$ & -0.19 & $0.3(1)$ & $0.035(42)$ \\
\hline$b^{\Lambda}$ & $-0.10(3)$ & $0.065(33)$ & $-0.14(6)$ & $-0.12(3)$ \\
\hline
\end{tabular}

